\title{
Electromigration Immortality Check considering Joule Heating Effect for Multisegment Wires*
}

\author{
Mohammadamir Kavousi ${ }^{1}$, Liang Chen $^{1}$ and Sheldon X.-D. Tan ${ }^{1}$ \\ ${ }^{1}$ Department of Electrical and Computer Engineering, University of California, Riverside, CA 92521 \\ mkavo003@ucr.edu,lianchen@ucr.edu,stan@ece.ucr.edu
}

\begin{abstract}
Electromigration (EM) is still the most important reliability concern for VLSI systems, especially at the nanometer regime. EM immortality check is an important step for full-chip EM signoff analysis. In this paper, we propose a new electromigration (EM) immortality check method for multi-segment interconnect considering the impacts of Joule heating induced temperature gradient. Temperature gradients from metal Joule heating, called thermal migration, can be a significant force for the metal atomic migrations, and these impacts get more significant as technology scales down. Compared to existing methods, the new method can consider the spatial temperature gradient due to Joule heating for multi-segment wires for the first time. We derive the analytic solution for the resulting steadystate EM-thermal migration stress distribution problem. Then we develop the new temperature-aware voltage-based EM immortality check method considering the multi-segment temperature migration effects, which carries all the benefits of the recently proposed voltage-based EM immortality method for multi-segment interconnects. Numerical results on an IBM power grid and self synthesized power delivery networks show that the proposed temperatureaware EM immortality check method is much more accurate than recently proposed state of the art EM immortality method.
\end{abstract}

\section{KEYWORDS}

Electromigration (EM), Joule heating, Steady-state solution, Multibranch interconnects, Immortality conditions

\section{ACM Reference Format:}

Mohammadamir Kavousi ${ }^{1}$, Liang Chen ${ }^{1}$ and Sheldon X.-D. Tan ${ }^{1}$. 2020. Electromigration Immortality Check considering Joule Heating Effect for Multisegment Wires. In IEEE/ACM International Conference on Computer-Aided Design (ICCAD '20), November 2-5, 2020, Virtual Event, USA. ACM, New York, NY, USA, 8 pages. https://doi.org/10.1145/3400302.3415634

\section{INTRODUCTION}

Due to the increasing current densities and decreasing dimensions of the interconnects, electromigration (EM) induced aging and failure effects remained the top reliability concerns for modern VLSI chips in 7-nm technology and below. Therefore, it is important

\footnotetext{
"This work is supported in part by NSF grants under No. CCF-1816361, in part by NSF grant under No. CCF-2007135 and No. OISE-1854276.
}

This work is licensed under a Creative Commons Attribution International 4.0 License. ICCAD '20, November 2-5, 2020, Virtual Event, USA

(C) 2020 Copyright held by the owner/author(s)

ACM ISBN 978-1-4503-8026-3/20/11.

https://doi.org/10.1145/3400302.3415634 to develop more accurate and less conservative EM sign-off and validation [1]. However, it is well accepted that the existing Black and Blech-based EM models are subject to growing criticism due to over conservativeness and they only work for a single wire segment [2, 3]. To mitigate the existing problem in EM models, a number of physics-based EM models and assessment techniques have been proposed $[1,4-16]$. Those EM models primarily focus on solving the partial differential equation (PDE) (called Korhonen's equation) of hydrostatic stress evolution in the confined multisegment wires subject to blocking material boundary conditions. However, most of those EM models do not consider the temperature gradient impacts on the metal migration process.

The continuing demand in ICs for smaller size and greater functionality increases current densities significantly while 3D stacked IC integration technique results in poor heat dissipation. Joule heating becomes a serious reliability issue. We usually use vias, Through-Silicon-Vias (TSVs), and Heterogeneous InterConnect (HIC) to provide efficient cooling paths for transferring the heat between different layers. Unfortunately, the cooling paths, in turn, lead to high temperature gradients. Also, different current densities of connected interconnects can result in large spatial temperature gradients. It has been experimentally observed that temperature gradient $|\mathrm{d} T / \mathrm{d} x|$ can easily exceed more than $0.1 \mathrm{~K} / \mu \mathrm{m}$ [16-20]. Also, experimental data shows that thermal gradient can have significant impacts on the EM-induced time to failure (TTF) for power electronics due to elevated temperature and large temperature gradients [21]. This work shows that the thermal gradient of $0.19 \mathrm{~K} / \mu \mathrm{m}$ can lead to $50 \%$ TTF reduction even with a low oven temperature. As a result, the temperature gradient effects can be quite significant (almost in the same magnitude as the current induced migration) due to the Joule heating effects. Hence, it is imperative to consider thermomigration (TM) effect due to significant Joule heating in modern ICs.

Some EM analysis research efforts have been carried out recently considering the transient/temporal thermal effects when solving the Korhonen's equation or derive analytic models to estimate transient EM stress under time-varying temperature [8, 10, 11, 22, 23]. Those works, however, did not consider the spatial temperature or thermal gradient impacts on the multi-segment wires.

For EM immortality check, which can be viewed as steady-state stress analysis, Blech's product [3] was a widely used method for industry. But it only works for a single wire segment, which can lead to significant over conservation. To mitigate this problem, recently a voltage-based $[9,14]$ has been proposed for multi-segment interconnect wires. However, this approach did not consider spatial temperature gradient impacts. 
Recently research works have been proposed to investigate EM considering the thermal immigration effects [16, 24-28]. Abbasinasab et al. proposed analytical solutions for steady states of the EM-TM model and extended voltage based EM immortality check to consider thermal migration effects [16, 27, 28]. However, the temperature of each segment from Joule heating is computed separately, which leads to accuracy loss as we show in this work. Also, no initial stress from thermal-mechanical stressing is considered in this work as well.

In this paper, we propose a new electromigration (EM) immortality check method for multi-segment interconnect considering the impacts of Joule heating induced temperature gradient for the first time. Our contributions are as follows:

- We show that Joule heating induced temperature has to be computed for all the wire segments, instead of individually computed for a single segment. Compared to [16], the new method takes the temperature distribution into consideration for multi-segment wires for the first time.

- We derive the semi-analytic solution to obtain the terminal temperatures of all the wire segments due to Joule heating.

- We develop the analytic expression to show the impacts of non-zero initial stress distributions on steady-state stress considering different temperature distributions.

- We derive the analytic solution for steady-state stress distributions considering different temperature distributions caused by Joule heating. Then we develop the new temperatureaware voltage-based EM immortality check method considering the multi-segment temperature distributions.

- Numerical results on an IBM power grid and self synthesized power delivery networks show that the proposed temperatureaware EM immortality check method is much more accurate than the recently proposed state of the art EM immortality method.

The paper is organized as follows: Section 2 reviews existing EM immortality check methods. Section 3 introduces the steadystate analytic solution for temperature distribution considering Joule heating and steady-state stress solution considering the temperature and initial non-zero stress distributions for a single wire segment. Then we extend the semi-analytic solution for steady-state stress distribution on a multi-segment wire in Section 4 . Section 5 derives the new temperature-aware voltage-based EM immortality check method. Numerical results are presented in Section 6. Finally, section 7 concludes this paper.

\section{REVIEW OF RELATED WORK}

EM immortality check is an important part of EM validation and sign off step in the design flow. The traditional method mainly focuses on the current density of each individual wire segment. Specifically, If the critical stress that the wire can withstand is $\sigma_{\text {crit }}$ and $\sigma_{\text {init }}$ is the initial stress, we can define the critical product for EM failure as

$$
(j L)_{c r i t}=\frac{\Omega\left(\sigma_{\text {crit }}-\sigma_{\text {init }}\right)}{e Z \rho}
$$

which is called the Blech limit or Blech product [3]. $\Omega$ is the atomic lattice volume, $e$ is the electron charge, $e Z$ is the effective charge of the migrating atoms, $\rho$ is the wire electrical resistivity and $j$ is the current density.

A wire is immortal for EM if it satisfies $j L<(j L)_{\text {crit }}$. As a result, the Blech product can help identify all the immortal wires efficiently.

However, the recent study shows that we have to consider all segment wires in an interconnect tree [1]. Sun et al recently proposed voltage-based immortality check for multi-segment interconnect $[9,14]$. Specifically, for a given arbitrary interconnect tree with $N$ nodes, assuming the voltage in node $i$ is $U_{i}$ and the ground node is $g$ and $U_{g}=0$, then the stress at the node $i$ can be computed as

$$
\sigma_{i}=\frac{e Z}{\Omega}\left(V_{E}-U_{i}\right)
$$

where $V_{E}$ is defined as the EM Voltage,

$$
V_{E}=\frac{1}{2 A} \sum_{k \neq g}^{N} a_{k} U_{k}
$$

where $A$ is the total area of the wire segments and $a_{k}$ is the total area of the branches connected to the node. Given the Critical EM Voltage, $V_{C r i t, E M}$, defined as

$$
V_{\text {crit }, E M}=\frac{\Omega}{Z e}\left(\sigma_{\text {crit }}-\sigma_{\text {init }}\right)
$$

then EM immortality check for node $i$ becomes

$$
V_{c r i t, E M}>V_{E}-U_{i}
$$

Since we only need to look at the node with the lowest voltage, the ground node or cathode node of the whole tree, as a result, (5) can be simplified to $V_{\text {crit,EM }}>V_{E}$. If this EM check fails, then transient EM analysis will be carried out to find the void location and the nucleation time.

However, this voltage-based method did not consider the Joule heating from the interconnects. Recently, Abbasinasab et al. considered the Joule heating effects and provided a voltage based model to perform EM reliability check [16, 27, 28]. In this method, the node voltage needs to be adjusted by the temperature of the wire segment:

$$
\begin{aligned}
U_{i}^{T} & =U_{i}-\frac{Q}{Z e} \ln \left(T_{i}\right) \\
V_{E}^{T} & =\frac{1}{A} \sum_{k \in B}\left(U_{0 k}-\frac{Q}{Z e} \ln \left(T_{0 k}\right)\right) A_{k}
\end{aligned}
$$

Where $Q$ is the specific heat of transport, $U_{0 k}$ and $T_{0 k}$ are the average voltage and temperature ${ }^{1}$ of two end nodes in the $k$ th line, respectively, $A_{k}$ is the area of the $k$ th line, $A$ is the total area of all lines, $U_{i}$ and $T_{i}$ are the voltage and temperature at node $i$, respectively, $V_{E}^{T}$ is the EM voltage and $U_{i}^{T}$ is the effective voltage considering the temperature at node $i . B$ is the set of all the branches or segments in the interconnect tree.

Given the same critical voltage definition in (4), then it gives the voltage-based EM immortality check formula

$$
V_{\text {crit }, E M}>V_{E}^{T}-U_{i}^{T}
$$

\footnotetext{
${ }^{1}$ Actually $T_{0 k}$ should be the geometrical average of temperature of two end nodes
} 
which is the same as (5). However, the temperature of each segment due to Joule heating is computed separately, which leads to accuracy loss as we show in this work.

\section{STEADY STATE ANALYTIC SOLUTIONS FOR TEMPERATURE AND STRESS FOR A SINGLE WIRE}

\subsection{Analytic solution for Joule heating induced temperature distribution}

We first estimate temperature distributions along the interconnects due to Joule heating. Heat conduction in VLSI circuits is described by the steady-state governing equation

$$
\nabla \cdot\left(-\kappa_{\mathrm{T}} \nabla T\right)=g
$$

where $T$ is the temperature, $\kappa_{\mathrm{T}}$ represents the material's thermal conductivity and $g$ is the power density of the heat source. For the heat source, we consider Joule heating and convective heat transfer, which are expressed as

$$
g=g_{j h}+g_{\text {Convection }}
$$

Joule heating effect is described by

$$
g_{j h}=\frac{I^{2} R}{V}=j^{2} \rho
$$

where $I$ is the current, $R$ is the resistance, $V$ is the volume of the heat source, $j$ is the current density and $\rho$ is the resistivity. For convective heat, we have

$$
\text { gConvection }=\frac{h}{l}\left(T_{0}-T\right)
$$

where $T_{0}$ is the ambient temperature, $h$ is the coefficient of heat transfer and $l$ is the thermal characteristic length.

Therefore, we can obtain the governing equation for the interconnects

$$
\nabla^{2} T-\frac{1}{\Gamma^{2}}\left(T-T_{0}\right)+\frac{j^{2} \rho}{\kappa_{\mathrm{T}}}=0
$$

where $\Gamma$ is called effective thermal length, which can be computed as

$$
\Gamma^{-2}=\frac{1}{\kappa_{\mathrm{T}}} \frac{h}{l}
$$

For the interconnects, we can reduce the $3 \mathrm{D}$ problem into $1 \mathrm{D}$ problem with the convective heat. The governing equation of $\mathrm{Cu}$ interconnects is given by

$$
\frac{\partial^{2} T}{\partial x^{2}}-\frac{1}{\Gamma^{2}}\left(T-T_{0}\right)+\frac{j^{2} \rho}{\kappa_{\mathrm{Cu}}}=0
$$

We want to stress that in our method the terminal temperature at the boundaries $T\left(-\frac{L}{2}\right)=T_{i}$ and $T\left(+\frac{L}{2}\right)=T_{j}$ does not bear any assumption and we will discuss how they are determined in Section 4 . In contrast, work in [16] assume that $\left(T_{i}+T_{j}\right) / 2=T_{0}$ so that temperature can be computed for individual wire separately, which can lead to significant accuracy loss as we show later.

Assume that $T_{i}$ and $T_{j}$ are known, then the solution of (14) is given by

$$
\begin{array}{r}
T(x)=\left(\frac{T_{i}+T_{j}}{2}-T_{m}-T_{0}\right) \operatorname{sech}\left(\frac{L}{2 \Gamma}\right) \cosh \left(\frac{x}{\Gamma}\right) \\
+T_{n} \operatorname{csch}\left(\frac{L}{2 \Gamma}\right) \sinh \left(\frac{x}{\Gamma}\right)+T_{m}+T_{0}
\end{array}
$$

where

$$
T_{m}=\frac{j^{2} \rho \Gamma^{2}}{\kappa_{\mathrm{Cu}}}, T_{n}=\frac{T_{i}-T_{j}}{2}
$$

Then, analytic solution (15) can be further re-written as:

$$
T(x)=A \cosh (a x)+B \sinh (a x)+C
$$

where

$$
\begin{aligned}
& a=\frac{1}{\Gamma} ; A=\left(\frac{T_{i}+T_{j}}{2}-T_{m}-T_{0}\right) \operatorname{sech}\left(\frac{L}{2 \Gamma}\right) \\
& B=T_{n} \operatorname{csch}\left(\frac{L}{2 \Gamma}\right) ; C=T_{m}+T_{0}
\end{aligned}
$$

With such notations, following the similar approximation: $|A| \ll$ $|C|,|B| \ll|C|$ [27], we can compute temperature log integral for (17) as follows:

$$
\begin{aligned}
\int_{-\frac{L}{2}}^{+\frac{L}{2}} \ln (T(x)) d x & =(\ln (C)) L+\frac{2}{a} \sinh \left(\frac{a L}{2}\right)\left(\frac{A}{C}\right) \\
& =\left(\ln \left(T_{m}+T_{0}\right)\right) L \\
& +\Gamma \tanh \left(\frac{L}{2 \Gamma}\right)\left(\frac{T_{i}+T_{j}-2\left(T_{m}+T_{0}\right)}{T_{m}+T_{0}}\right)
\end{aligned}
$$

Now we have to determine the terminal temperature $T_{i}$ and $T_{j}$ to compute $\int_{-\frac{L}{2}}^{+\frac{L}{2}} \ln (T(x)) d x$, which to be presented in Section 4 .

\subsection{Analytic solution for stress under temperature gradient and initial residual stress distribution}

Now, let's compute the steady-state stress of a segment under the given temperature distribution for the single segment. The steady state of a system under material migration is achieved when all atomic fluxes cancel each other out, which mathematically means

$$
J_{a}=\kappa(x)\left(\frac{\partial \sigma(x, \infty)}{\partial x}-\frac{e Z \rho j}{\Omega}-\frac{Q}{\Omega T} \frac{\partial T}{\partial x}\right)=0
$$

By integration of (20), we have

$$
\sigma(x, \infty)=\frac{e Z \rho j}{\Omega} x+\frac{Q}{\Omega} \ln (T(x))+\text { constant }
$$

To eliminate the constant value, the difference stress between two end-points is given by

$$
\sigma\left(x_{i}\right)-\sigma\left(x_{j}\right)=\frac{e Z \rho j}{\Omega} L_{i j}+\frac{Q}{\Omega}\left[\ln \left(T\left(x_{i}\right)\right)-\ln \left(T\left(x_{j}\right)\right)\right]
$$

By integration of (21) we have[27]:

$$
\begin{aligned}
\int_{-\frac{L}{2}}^{+\frac{L}{2}} \sigma(x) d x & =\frac{\sigma_{i}+\sigma_{j}}{2} L-\frac{Q}{\Omega} \frac{\ln T_{i}+\ln T_{j}}{2} L \\
& +\frac{Q}{\Omega} \int_{-\frac{L}{2}}^{+\frac{L}{2}} \ln T(x) d x \\
& =\frac{\sigma\left(x_{i}\right)+\sigma\left(x_{j}\right)}{2} L_{i j}+\frac{Q}{\Omega}\left(\ln \frac{T_{m}+T_{0}}{\sqrt{T_{i} T_{j}}} L_{i j}\right. \\
& \left.+\Gamma \tanh \left(\frac{L_{i j}}{2 \Gamma}\right)\left(\frac{T_{i}+T_{j}-2\left(T_{m}+T_{0}\right)}{T_{m}+T_{0}}\right)\right)
\end{aligned}
$$

This is an important equation to show how the temperature will impact on the steady-state stress distribution for a single wire. 


\section{STEADY STATE STRESS SOLUTION FOR A MULTI-SEGMENT INTERCONNECT}

In a practical VLSI circuits layout, the structures of interconnects are more complex, such as star-like tree, straight line, and general multi-segment interconnect tree. The estimation of steady temperature and stress for those complex branches is not independent and should be considered simultaneously. To consider the connected effects of general multi-segment interconnects, we should satisfy the continuity of atom flux (heat flux) and stress (temperature) at the junction node. In this section, we extend a single wire to a general multi-segment interconnect.

\subsection{Analytic temperature solution for a multi-segment wire}

For general multi-segment wires, we assume that each wire segment $i j$ has a direction defined for the sign of its heat flux $\mathrm{q}_{i j}(x)$ at the interior junction nodes $x_{r} \in\left\{x_{r 1}, x_{r 2}, \ldots, x_{r p}\right\}$. Then we observe that heat flux conservation is satisfied at location $x_{r}$, which is described by

$$
\sum_{i j} \mathbf{q}_{i j}\left(x_{r}\right)=0
$$

We note that this heat flux conservation ignores the heat loss to the surrounding dielectrics at $x_{r}$, which we assume is very small in general.

At the block terminals $x_{b} \in\left\{x_{b 1}, x_{b 2}, \ldots, x_{b q}\right\}$, heat flux flows from the terminal to its surrounding dielectrics due to the convective boundary condition, which is modeled by

$$
-\mathbf{q}_{i j}\left(x_{b}\right) \cdot \mathbf{n}_{b}=h_{b}\left(T_{i j}\left(x_{b}\right)-T_{0}\right)
$$

where $h_{b}$ is the convective coefficient. The heat flux and temperature are related by Fourier's law, which is expressed as

$$
\mathrm{q}=-\kappa_{\mathrm{Cu}} \nabla T
$$

Therefore, substituting (26) to boundary conditions (24) and (25), we have

$$
\begin{aligned}
& B C:\left.\sum_{i j} \frac{\partial T_{i j}(x)}{\partial x}\right|_{x=x_{r}} \cdot n_{r}=0 \\
& B C:\left.\frac{\partial T_{i j}(x)}{\partial x}\right|_{x=x_{b}} \cdot n_{b}=\Gamma_{b}^{-2}\left(T_{i j}\left(x_{b}\right)-T_{0}\right)
\end{aligned}
$$

where the terminal thermal length is given by

$$
\Gamma_{b}=\sqrt{\frac{\kappa_{\mathrm{Cu}}}{h_{b}}}
$$

Finally, based on boundary conditions (27), temperature expression (15) and its derivative, we can form the linear equations

$$
\mathrm{A} \cdot \boldsymbol{\beta}=\mathrm{B}
$$

where $\mathbf{A}$ is an $(p+q) \times(p+q)$ matrix, $\mathbf{B}$ is an $(p+q) \times 1$ vector and the vector to be determined is represented by

$$
\boldsymbol{\beta}=\left[T_{i j}\left(x_{1}\right), T_{i j}\left(x_{2}\right), \cdots, T_{i j}\left(x_{n}\right)\right]^{T}
$$

The elements of matrix A are connected with a tree structure, which can be solved linearly and efficiently. Once the temperature of $n$ nodes is obtained, temperature distribution along the interconnects can be determined by (15).
We notice that at the terminal nodes (vias typically), the related elements of $\boldsymbol{\beta}$ are known already, which are called $\boldsymbol{\beta}_{d}$. To solve this problem, we reorder and decompose equation (29), which is expressed as

$$
\left[\begin{array}{ll}
\mathbf{A}_{11} & \mathbf{A}_{12} \\
\mathbf{A}_{21} & \mathbf{A}_{22}
\end{array}\right] \cdot\left[\begin{array}{l}
\boldsymbol{\beta}_{u} \\
\boldsymbol{\beta}_{d}
\end{array}\right]=\left[\begin{array}{l}
\mathbf{B}_{1} \\
\mathbf{B}_{2}
\end{array}\right]
$$

where $\boldsymbol{\beta}_{u}$ denotes unknown elements of $\boldsymbol{\beta}$ and $\boldsymbol{\beta}_{d}$ represents Dirichlet boundary elements of $\boldsymbol{\beta}$. Therefore, the unknown temperature can be calculated by

$$
\boldsymbol{\beta}_{u}=\mathrm{A}_{11}^{-1}\left(\mathrm{~B}_{1}-\mathrm{A}_{12} \cdot \boldsymbol{\beta}_{d}\right)
$$

\subsection{Analytic stress solution for a multi-segment wire considering the initial stress distribution}

For general multi-segment wires, stress also need to satisfy the atom conservation. Based on (23), the integration of stress for all branches can be expressed by

$$
\begin{aligned}
\int \sigma(x) d x & =\sum_{k \in B} \int_{-\frac{L_{k}}{2}}^{+\frac{L_{k}}{2}} \sigma_{k}(x) d x \\
& =\sum_{i j} \int_{x_{i}}^{x_{j}} \sigma(x) d x \\
& =\sum_{i j}\left[\frac{\sigma_{i}+\sigma_{j}}{2} L_{i j}+\frac{Q}{\Omega}\left(\ln \left(\frac{T_{0}+T_{m, i j}}{\sqrt{T_{i} T_{j}}}\right) L_{i j}\right.\right. \\
& \left.\left.+\frac{\left(T_{i}+T_{j}-2\left(T_{0}+T_{m, i j}\right)\right) \Gamma}{\left(T_{0}+T_{m, i j}\right)} \tanh \left(\frac{L_{i j}}{2 \Gamma}\right)\right)\right]
\end{aligned}
$$

Now we can consider the impacts of initial stress on the steadystate stress distribution under Joule heating temperature. Based on Hooke's law, to satisfy the atom conservation, we have

$$
\int \sigma(x) d x=\int \sigma_{0} d x
$$

where $\sigma_{0}(x)$ is the initial stress distribution. Then we have

$$
\begin{aligned}
& \sum_{i j}\left[\frac{\sigma_{i}+\sigma_{j}}{2} L_{i j}+\frac{Q}{\Omega}\left(\ln \left(\frac{T_{0}+T_{m, i j}}{\sqrt{T_{i} T_{j}}}\right) L_{i j}\right.\right. \\
& \left.\left.+\frac{\left(T_{i}+T_{j}-2\left(T_{0}+T_{m, i j}\right)\right) \Gamma}{\left(T_{0}+T_{m, i j}\right)} \tanh \left(\frac{L_{i j}}{2 \Gamma}\right)\right)\right]=\int \sigma_{0}(x) d x
\end{aligned}
$$

With (22) and (35), we can calculate the steady stress for the general multi-segment interconnects.

\section{NEW TEMPERATURE-AWARE VOLTAGE BASED EM IMMORTALITY CHECK METHOD}

Based on Ohm's Law, the voltage of a two-node wire segment can be expressed as

$$
U_{i j}=U_{i}-U_{j}=I R_{i j}=j S \times \rho \frac{L_{i j}}{S}=j \rho L_{i j}
$$

where $U_{i j}$ is the voltage between node $i$ and node $j, U_{i}$ and $U_{j}$ are the potentials at node $i$ and $j$, respectively, and $S$ is the cross area of the interconnects. 
One important observation is that the stress difference between two nodes at the steady-state can be expressed in terms of voltage, instead of current density [29]. Based on (21), such observation can still be applied to the situation under different temperature:

$$
\begin{aligned}
\sigma_{i}-\sigma_{j} & =\frac{e Z}{\Omega} j \rho L_{i j}+\frac{Q}{\Omega}\left(\ln \left[T\left(x_{i}\right)\right]-\ln \left[T\left(x_{j}\right)\right]\right) \\
& =\frac{e Z}{\Omega} U_{j i}+\frac{Q}{\Omega}\left(\ln \left[T\left(x_{i}\right)\right]-\ln \left[T\left(x_{j}\right)\right]\right)
\end{aligned}
$$

With the node $g$ as the ground node, then $U_{i g}=U_{i}$, and $U_{j g}=U_{j}$, the stress at node $i$ and $j$ can be given by

$$
\begin{gathered}
\sigma_{i}=\sigma_{g}-\frac{e Z}{\Omega} U_{i}-\frac{Q}{\Omega}\left(\ln \left[T\left(x_{g}\right)\right]-\ln \left[T\left(x_{i}\right)\right]\right) \\
\sigma_{j}=\sigma_{g}-\frac{e Z}{\Omega} U_{j}-\frac{Q}{\Omega}\left(\ln \left[T\left(x_{g}\right)\right]-\ln \left[T\left(x_{j}\right)\right]\right)
\end{gathered}
$$

By substituting (38) into (34), we have

$$
\begin{aligned}
\sigma\left(x_{g}\right)- & \frac{Q}{\Omega} \ln \left(T\left(x_{g}\right)\right)= \\
& \frac{1}{\sum_{i j} L_{i j}} \sum_{i j} \frac{\left(U_{i}+U_{j}\right)}{2} \frac{e Z}{\Omega} L_{i j} \\
& +\frac{1}{\sum_{i j} L_{i j}}\left[\int \sigma_{0}(x) d x\right. \\
& -\sum_{i j}\left[\frac { Q } { \Omega } \left(\ln \left(T_{0}+T_{m}\right) L_{i j}\right.\right. \\
& \left.\left.\left.+\frac{\left(T_{i}+T_{j}-2\left(T_{0}+T_{m}\right)\right) \Gamma}{\left(T_{0}+T_{m}\right)} \tanh \left(\frac{L_{i j}}{2 \Gamma}\right)\right)\right]\right]
\end{aligned}
$$

Then we can define a new temperature-aware EM voltage considering Joule heating induced temperature distribution of all the wire segments as

$$
\begin{aligned}
V_{E}^{T} & =\frac{1}{\sum_{i j} L_{i j}} \sum_{i j} \frac{U_{i}+U_{j}}{2} L_{i j} \\
& +\frac{1}{\sum_{i j} L_{i j}} \frac{\Omega}{e Z}\left[\int \sigma_{0}(x) d x\right. \\
& -\sum_{i j}\left[\frac { Q } { \Omega } \left(\ln \left(T_{0}+T_{m}\right) L_{i j}\right.\right. \\
& \left.\left.\left.+\frac{\left(T_{i}+T_{j}-2\left(T_{0}+T_{m}\right)\right) \Gamma}{\left(T_{0}+T_{m}\right)} \tanh \left(\frac{L_{i j}}{2 \Gamma}\right)\right)\right]\right]
\end{aligned}
$$

Then, the steady stress at the node $k$ can be calculated as

$$
\sigma_{k}-\frac{Q}{\Omega} \ln \left(T_{k}\right)=\frac{e Z}{\Omega}\left(V_{E}^{T}-U_{k}\right)
$$

where $T_{k}$ is the temperature at node $k$. If we define critical $E M$ voltage as $V_{c r i t, E M}^{T}=\frac{\Omega}{Z e} \sigma_{c r i t}$, for any nodal voltage $V_{i}$, we can derive the same voltage based EM immortality check

$$
\begin{aligned}
V_{c r i t, E M}^{T} & >V_{E}^{T}-U_{k}+\frac{Q}{e Z} \ln \left(T_{k}\right) \text { or } \\
V_{c r i t, E M}^{T} & >V_{E}^{T}-U_{k}^{T}
\end{aligned}
$$

which is the same as (5), where $U_{K}^{T}$ is defined in (6). If this condition is met for all the terminal voltages of the wire, then no EM failures will happen. Therefore, we define a new Effective EM Voltage for node $k$

$$
V_{\mathrm{eff}, \mathrm{k}}=V_{E}^{T}-U_{k}+\frac{Q}{e Z} \ln \left(T_{k}\right) \text { or } V_{E}^{T}-U_{k}^{T}
$$

Then the stress at the node $k \sigma_{k}$ can be computed as

$$
\sigma_{k}=\frac{e Z}{\Omega} V_{\text {eff, } \mathrm{k}} \text { or } \sigma_{k}=\frac{e Z}{\Omega}\left(V_{E}^{T}-U_{k}^{T}\right)
$$

Typically, we only need to check the ground node or cathode node as it has the largest EM voltage, which means if $V_{E}^{T}=<$ $V_{c r i t, E M}^{T}$, then no wire will have an EM failure. This makes the voltage-based immortality check much more efficient as we typically only need to check one node for an interconnect segment, instead of checking every branch as is done for the traditional method. Note that if we have multiple nodes failing the check (43), all those nodes may lead to nucleation. However, as far as EM immortality is concerned, as long as one node fails as per (43), the whole tree is mortal.

\section{NUMERICAL RESULTS AND DISCUSSIONS}

In this section, we present some numerical results and validate the accuracy of the proposed analytical method with two typical structures in VLSI interconnects, including a straight line and multisegment interconnects. We also compare the proposed method against the recently proposed thermal-aware steady-state EM analysis method, called RAIN [16] for the two examples. All programs are implemented in MATLAB and tested on a standard computer.

\subsection{A straight line wire of power grid networks}

We will first validate our proposed method using the straight line interconnects, which comes from the industry IBMPG benchmarks [30]. Fig. 1(a) shows the IBMPG benchmark and Fig. 1(b) shows one of the interconnect tree.

As an example, the segment sizes and current densities of each interconnect wire obtained by SPICE circuits simulation for IBMPG1 benchmark shown in Fig. 1(b) are demonstrated in Table 1. Where $\mathrm{Br}$ is the branch index, $j$ is the current density and $L$ is the length of the wire.

\begin{tabular}{||c|c|c|c|c|c||}
\hline $\mathrm{Br}$ & $\mathrm{j}\left(A / m^{2}\right)$ & $\mathrm{L}(\mu \mathrm{m})$ & $\mathrm{Br}$ & $\mathrm{j}\left(A / m^{2}\right)$ & $\mathrm{L}(\mu \mathrm{m})$ \\
\hline \hline 1 & $-1.0565 \times 10^{10}$ & 11 & 6 & $-4.4610 \times 10^{10}$ & 119 \\
\hline 2 & $-5.0893 \times 10^{9}$ & 119 & 7 & $-5.7618 \times 10^{10}$ & 11 \\
\hline 3 & $-4.7170 \times 10^{10}$ & 11 & 8 & $2.2159 \times 10^{10}$ & 119 \\
\hline 4 & $-4.4349 \times 10^{10}$ & 119 & 9 & $1.8564 \times 10^{10}$ & 11 \\
\hline 5 & $-4.4133 \times 10^{10}$ & 11 & & & \\
\hline
\end{tabular}

Table 1: Parameters for the straight line interconnects

For the straight line case, we found the temperature at nodes by applying 1-D data interpolation function to the COMSOL results. Also for the multi-segment interconnects, We set constant temperatures at nodes 2 and 16 with $373 \mathrm{~K}$ and $393 \mathrm{~K}$, respectively. In this case, the temperatures at all nodes are estimated by solving the equations in (32).

Fig. 2(a) shows the temperature profile across the straight line. Once temperature distribution along the interconnect is captured, 


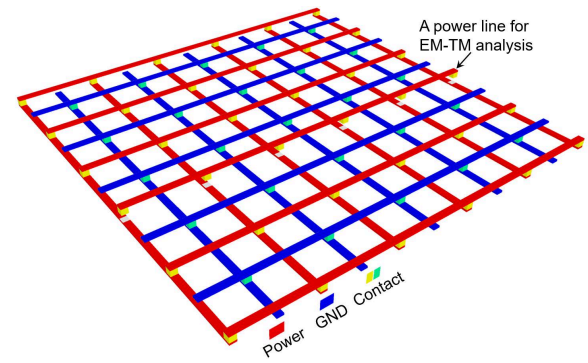

(a)

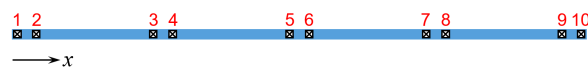

(b)

Figure 1: (a) The structure of a typical power delivery network. We can evaluate EM stress separately for each power line (red) because the diffusion barrier (Ta/TaN) prevents $\mathrm{Cu}$ atoms from diffusing into other metal layers. (b) A straight line structure extracted from IBMPG1 benchmark [30], which is drawn from real design. Positive value of current density denotes its direction is along $x$ positive direction.

we can determine the steady-state hydrostatic stress. Fig. 3 show the comparison results in terms of effective voltage (44) against the COMSOL, COMSOL with constant temperature and the RAIN method [16]. As we can see, the proposed method match with COMSOL almost perfectly. While the RAIN and COMSOL with constant temperature show clearly discrepancy with the COMSOL results.

\subsection{A multi-segment wire in standard cells}

The second type of interconnects is multi-segment power interconnects of a standard cell for designing ICs. As we can see, the power line (red) is a tree structure and has multi-segments. Therefore, a more complicated and general multi-segment wire structure as shown in Fig. 4 is employed as a test case. As we can see in this figure, we assumed a general multi-segment wire with several branches to show the authenticity of our method for different benchmarks. The current densities and lengths of all the segments are given in Table 2, where $\mathrm{Br}$ is the branch index, $j$ is the current density, and $L$ is the length of the wire.

As we can see that the results from the proposed method are very accurate compared to the results of COMSOL as shown in Fig. 3 and Fig. 5. These figures also show that the analytical solution of steadystate in [16] is less accurate in the multi-segment interconnect case. Then obtained effective voltage (44) is more accurate than recently proposed EM immortality method and it helps us to better determine whether or not the EM failure will happen.

Fig. 5 shows the comparison results in terms of effective voltage (44) against the COMSOL, COMSOL with constant temperature and the RAIN method again. We show the comparison results for two different initial conditions. As we can see, the proposed method

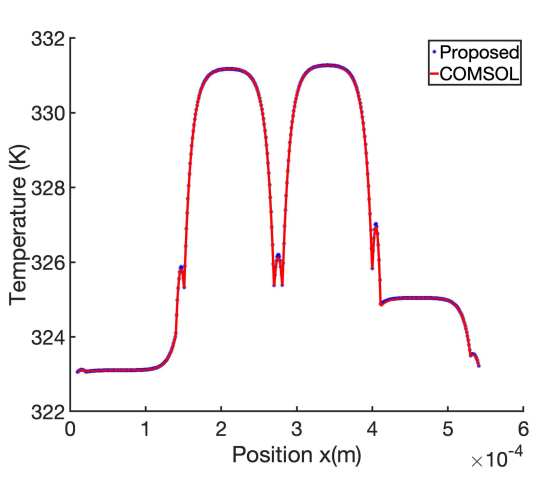

(a)

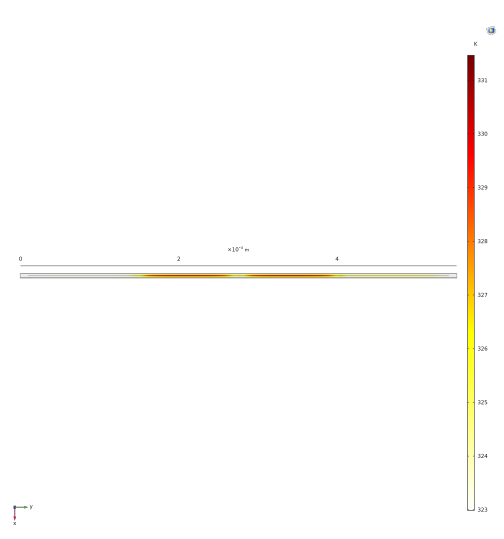

(b)

Figure 2: Temperature distribution across a straight line interconnect.

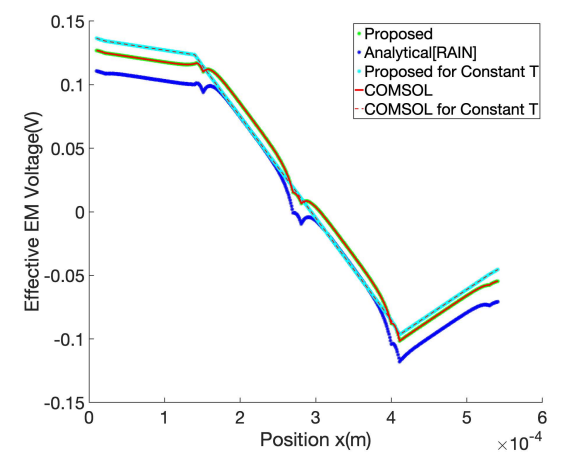

Figure 3: Effective EM voltage of a straight line interconnect.

match with COMSOL almost perfectly. While the RAIN and COMSOL with constant temperature show clearly discrepancy with the COMSOL results.

Fig. 6(a) shows the EM voltage of a multi-segment interconnects with different initial stresses. The EM voltage can offset with different initial stresses and its curve keeps the same. The EM voltage offset due to the initial stresses is: 


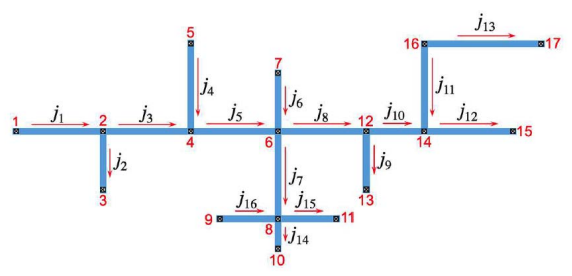

(a)

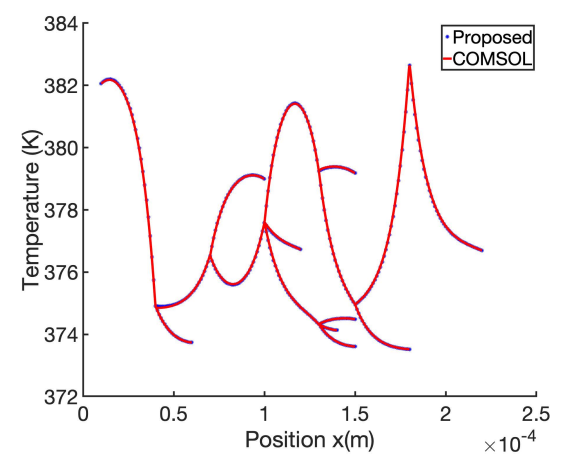

(b)

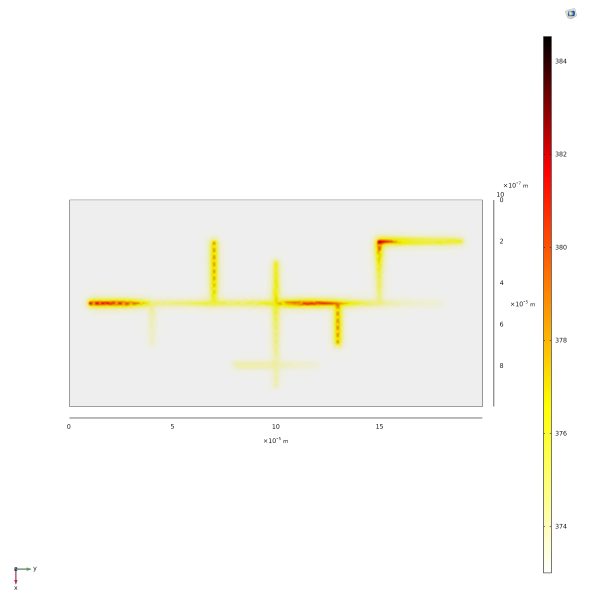

(c)

Figure 4: (a) Structure and (b), (c) temperature distributions of a multi-segment interconnect

$$
V_{\mathrm{eff}}^{\mathrm{Offset}}=\frac{\Omega}{Z e} \frac{1}{\sum_{i j} L_{i j}} \int \sigma_{0}(x) d x
$$

And from (45) we can find the stress offset because of considering the initial stresses:

$$
\sigma^{\text {Offset }}=\frac{1}{\sum_{i j} L_{i j}} \int \sigma_{0}(x) d x
$$

\begin{tabular}{||c|c|c|c|c|c||}
\hline $\mathrm{Br}$ & $\mathrm{j}\left(A / m^{2}\right)$ & $\mathrm{L}(\mu \mathrm{m})$ & $\mathrm{Br}$ & $\mathrm{j}\left(A / m^{2}\right)$ & $\mathrm{L}(\mu \mathrm{m})$ \\
\hline \hline 1 & $5 \times 10^{10}$ & 30 & 9 & $4 \times 10^{10}$ & 20 \\
\hline 2 & $1 \times 10^{10}$ & 20 & 10 & $2 \times 10^{10}$ & 20 \\
\hline 3 & $2 \times 10^{10}$ & 30 & 11 & $2 \times 10^{10}$ & 30 \\
\hline 4 & $4 \times 10^{10}$ & 30 & 12 & $1 \times 10^{10}$ & 30 \\
\hline 5 & $2 \times 10^{10}$ & 30 & 13 & $3 \times 10^{10}$ & 40 \\
\hline 6 & $3 \times 10^{10}$ & 20 & 14 & $1.5 \times 10^{10}$ & 10 \\
\hline 7 & $2 \times 10^{10}$ & 30 & 15 & $1 \times 10^{10}$ & 20 \\
\hline 8 & $5 \times 10^{10}$ & 30 & 16 & $2 \times 10^{10}$ & 20 \\
\hline
\end{tabular}

Table 2: Parameters for the multi-segment interconnect wires

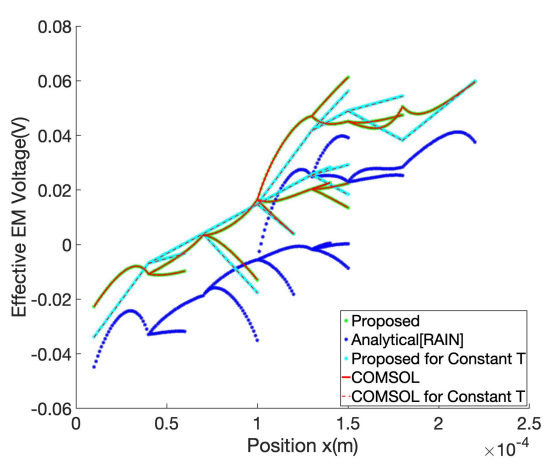

Figure 5: Effective EM voltage of a multi-segment interconnect.

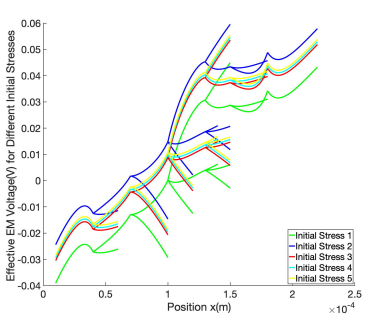

(a)

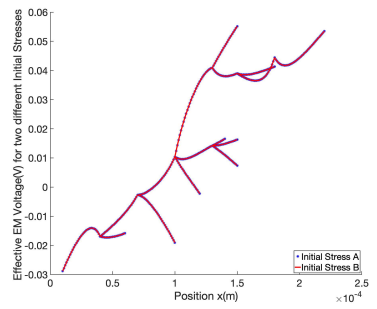

(b)
Figure 6: Effective EM voltage of a multi-segment interconnect with (a) different initial stresses and (b) the same values of initial stress integration $\int \sigma_{0} d x$.

We call this stress offset Effective Initial Stress. We can assume three types of functions for initial stresses and find the corresponding Effective Initial Stress to demonstrate the effects of initial stress on stress.

Constant Initial Stresses. In the simplest case to see the impacts of initial stress we assume $\sigma_{0}(x)=\sigma_{0}$, where $\sigma_{0}$ is a constant then we can obtain Effective Initial Stress as:

$$
\sigma_{0, \mathrm{eff}}=\sigma_{0}
$$


Step-Wise Initial Stresses. In a more realistic case, we can assume that each wire has constant initial stress that differs from the other wires. In this case, Effective Initial Stress is the weighted arithmetic mean of the initial stresses where weights are the length of wires:

$$
\sigma_{0, \mathrm{eff}}=\frac{\sum_{i j} L_{i j} \sigma_{0, i j}}{\sum_{i j} L_{i j}}
$$

Continuous Initial Stresses. In a realistic model, initial stresses are continuous and dependent on location $(x)$. Effective Initial Stress can be written as:

$$
\sigma_{0, \mathrm{eff}}=\frac{1}{L} \int_{0}^{L} \sigma_{0}(x) d x
$$

where $L=\sum_{i j} L_{i j}$. As we remember the Mean Value Theorem for Integrals, also in this case, Effective Initial Stress is the mean value of the initial stresses on $[0, L]$.

Another interesting fact about initial stresses is that we can have the same EM voltages and stresses for different initial stresses in an interconnect. If the values of initial stress integration $\int \sigma_{0} d x$ are the same, the EM voltage will keep the same even though initial stresses are different, as shown in Fig. 6(b).

\section{CONCLUSION}

In this paper, we have proposed a new voltage-based EM immortality check method considering the Joule heating induced spatial temperature gradient effects for general multi-segment interconnects for the first time. We essentially solved a steady-state solution for the eletromigration (EM) hydrostatic stress evolution considering thermomigration (TM) effects on general multi-segment interconnects based on voltage. We first derived the analytic solution to the resulting steady-state EM-thermal migration stress distribution problem. Then we developed the new temperature-aware voltagebased EM immortality check method considering the multi-segment temperature migration effects. Numerical results on an IBM power grid and self synthesized power delivery networks showed that compared to the COMSOL, our proposed temperature-aware EM immortality check method is more accurate than recently proposed state of the art EM immortality method. We have shown that the results of our method are almost the same as the COMSOL results while our analytical method is much more efficient than COMSOL.

\section{REFERENCES}

[1] S. X.-D. Tan, M. Tahoori, T. Kim, S. Wang, Z. Sun, and S. Kiamehr, VLSI Systems Long-Term Reliability - Modeling, Simulation and Optimization. Springer Publishing, 2019.

[2] J. R. Black, "Electromigration-A Brief Survey and Some Recent Results," IEEE Trans. on Electron Devices, vol. 16, no. 4, pp. 338-347, 1969.

[3] I. A. Blech, "Electromigration in thin aluminum films on titanium nitride," fournal of Applied Physics, vol. 47, no. 4, pp. 1203-1208, 1976

[4] X. Huang, T. Yu, V. Sukharev, and S. X.-D. Tan, "Physics-based electromigration assessment for power grid networks," in Proc. Design Automation Conf. (DAC). IEEE, June 2014, pp. 1-6.

[5] V. Sukharev, X. Huang, H.-B. Chen, and S. X.-D. Tan, "IR-drop based electromigration assessment: parametric failure chip-scale analysis," in Proc. Int. Conf. on Computer Aided Design (ICCAD). IEEE, Nov. 2014, pp. 428-433.

[6] H. Chen, S. X.-D. Tan, X. Huang, T. Kim, and V. Sukharev, "Analytical modeling and characterization of electromigration effects for multibranch interconnect trees," IEEE Trans. on Computer-Aided Design of Integrated Circuits and Systems, vol. 35, no. 11, pp. 1811-1824, 2016

[7] X. Huang, A. Kteyan, S. X.-D. Tan, and V. Sukharev, "Physics-based electromigration models and full-chip assessment for power grid networks," IEEE Trans. on Computer-Aided Design of Integrated Circuits and Systems, vol. 35, no. 11, pp. 1848-1861, Nov. 2016.

[8] V. Mishra and S. S. Sapatnekar, "Predicting Electromigration Mortality Under Temperature and Product Lifetime Specifications," in Proc. Design Automation Conf. (DAC), Jun. 2016, pp. 1-6.

[9] Z. Sun, E. Demircan, M. D. Shroff, T. Kim, X. Huang, and S. X.-D. Tan, "VoltageBased Electromigration Immortality Check for General Multi-Branch Interconnects," in Proc. Int. Conf. on Computer Aided Design (ICCAD), Nov. 2016, pp. 1-7.

[10] X. Huang, V. Sukharev, T. Kim, and S. X.-D. Tan, "Electromigration recovery modeling and analysis under time-depdendent current and temperature stressing," in Proc. Asia South Pacific Design Automation Conf. (ASPDAC). IEEE, Jan. 2016, pp. 244-249.

[11] X. Huang, V. Sukharev, and S. X.-D. Tan, "Dynamic electromigration modeling for transient stress evolution and recovery under time-dependent current and temperature stressing," Integration, the VLSI fournal, vol. 55, pp. 307-315, September 2016.

[12] S. Chatterjee, V. Sukharev, and F. N. Najm, "Power Grid Electromigration Checking using Physics-Based Models," IEEE Trans. on Computer-Aided Design of Integrated Circuits and Systems, vol. 37, no. 7, pp. 1317-1330, 2018.

[13] S. X.-D. Tan, H. Amrouch, T. Kim, Z. Sun, C. Cook, and J. Henkel, "Recent advances in EM and BTI induced reliability modeling, analysis and optimization," Integration, the VLSI fournal, vol. 60, pp. 132-152, Jan. 2018.

[14] Z. Sun, E. Demircan, M. D. Shroff, C. Cook, and S. X.-D. Tan, "Fast Electromigration Immortality Analysis for Multisegment Copper Interconnect Wires," IEEE Trans. on Computer-Aided Design of Integrated Circuits and Systems, vol. 37, no. 12, pp. 3137-3150, 2018.

[15] H. Zhao and S. X.-D. Tan, "Postvoiding fem analysis for electromigration failure characterization," IEEE Trans. on Very Large Scale Integration (VLSI) Systems, vol. 26, no. 11, pp. 2483-2493, Nov. 2018.

[16] A. Abbasinasab and M. Marek-Sadowska, "RAIN: A tool for reliability assessment of interconnect networks-physics to software," in Proc. Design Automation Conf. (DAC). New York, NY, USA: ACM, 2018, pp. 133:1-133:6.

[17] R. Oriani, "Thermomigration in solid metals," fournal of Physics and Chemistry of Solids, vol. 30, no. 2, pp. 339 - 351, 1969. [Online]. Available: http://www.sciencedirect.com/science/article/pii/0022369769903151

[18] Guo Weiling, Li Zhiguo, Zhou Tianyi, Cheng Yaohai, Chan Changhua, and Sheng Guangdi, "The electromigration and reliability of VLSI metallization under temperature gradient conditions," in 1998 5th International Conference on Solid-State and Integrated Circuit Technology. Proceedings (Cat. No.98EX105), Oct 1998, pp. 226-229.

[19] M. Li, D. W. Kim, S. Gu, D. Y. Parkinson, H. Barnard, and K. N. Tu, "Joule heating induced thermomigration failure in un-powered microbumps due to thermal crosstalk in 2.5d ic technology," Journal of Applied Physics, vol. 120, no. 7, p. $075105,2016$.

[20] K. N. Tu and A. M. Gusak, "A unified model of mean-time-to-failure for electromigration, thermomigration, and stress-migration based on entropy production," Journal of Applied Physics, vol. 126, no. 7, p. 075109, 2019.

[21] H. V. Nguyen, C. Salm, B. Krabbenborg, K. Weide-Zaage, J. Bisschop, A. J. Mouthaan, and F. G. Kuper, "Effect of thermal gradients on the electromigration life-time in power electronics," in 2004 IEEE International Reliability Physics Symposium. Proceedings, April 2004, pp. 619-620.

[22] Z. Lu, W. Huang, M. R. Stan, K. Skadron, and J. Lach, "Interconnect Lifetime Prediction for Reliability-Aware Systems," IEEE Transactions on Very Large Scale Integration (VLSI) Systems, vol. 15, no. 2, pp. 159-172, Feb 2007.

[23] H.-B. Chen, S. X.-D. Tan, J. Peng, T. Kim, and J. Chen, "Analytical modeling of electromigration failure for vlsi interconnect tree considering temperature and segment length effects," IEEE Transaction on Device and Materials Reliability (T-DMR), vol. 17, no. 4, pp. 653-666, 2017.

[24] R. De Orio, H. Ceric, and S. Selberherr, "Physically based models of electromigration: From black's equation to modern tcad models," Microelectronics Reliability, vol. 50, no. 6, pp. 775-789, 2010.

[25] X. Zha, "Numerical analysis of lead-free solder joints: Effects of thermal cycling and electromigration," Ph.D. dissertation, Loughborough University, March 2016.

[26] J. Xu, "Electromigration simulation of interconnection in very large scare integration," Ph.D. dissertation, Binghamton University, May 2016.

[27] A. Abbasinasab, "Interconnect aging-physics to software," Ph.D. dissertation, University of California Santa Barbara, June 2018.

[28] A. Abbasinasab and M. Marek-Sadowska, "Non-uniform temperature distribution in interconnects and its impact on electromigration," in Proceedings of the 2019 on Great Lakes Symposium on VLSI, ser. GLSVLSI '19. New York, NY, USA: ACM, 2019, pp. $117-122$.

[29] E. Demircan and M. D.Shroff, "Model based method for electro-migration stress determination in interconnects," in 2014 IEEE International Reliability Physics Symposium, June 2014, pp. IT.5.1-IT.5.6.

[30] S. R. Nassif, "Power Grid Analysis Benchmarks," in Proc. Asia South Pacific Design Automation Conf. (ASPDAC), Mar. 2008, pp. 376-381. 